\title{
Uma breve história da Computação Aplicada no Brasil
}

\author{
Adelaine Gelain ${ }^{1}$ \\ Ana Teruko Yokomizo Watanabe ${ }^{1}$ \\ Marina Silva Fouto ${ }^{1}$ \\ Rafaela Bosse ${ }^{1}$ \\ Marcelo da Silva Hounsell ${ }^{1}$
}

\begin{abstract}
Resumo: A área de pesquisa da Computação Aplicada (CA) no Brasil, em fase de consolidação, tem crescido em importância graças à sua relação com outras áreas. Por sua "jovialidade", pouco se tem analisado sobre a forma como se faz pesquisas nessa área. A Revista Brasileira de Computação Aplicada (RBCA) é uma publicação representativa desse foco que foi criada há pouco tempo. Então, para se ter uma visão das pesquisas na área de CA analisou-se a produção registrada na RBCA. Três análises foram feitas: quanto aos paradigmas científicos predominantes, estilo de maturidade e abordagem de pesquisa das publicações dos últimos cinco anos. Um checklist foi concebido para facilitar a análise mais subjetiva - do paradigma. Todos os artigos foram avaliados ao menos duas vezes, sendo que a maioria sofreu três avaliações distintas. As 501 avaliações em três rodadas permitiu caracterizar a produção como majoritariamente tecnocrática, usando estilos de maturidade iniciais e abordagens desenvolvimentista e descritiva. Esses elementos sugerem que os pesquisadores da área adotam o viés das Engenharias, o que é razoável considerando o aspecto "aplicado". Entretanto, para a CA consolidar-se como uma ramificação da Ciência da Computação (CC), vê-se importante que os pesquisadores da CA utilizem metodologias científicas de maior credibilidade.
\end{abstract}

Palavras-chave: Computação aplicada. Metodologia da pesquisa. Metodologia científica.

\begin{abstract}
The Applied Computing (AC) field of research in Brazil, yet to become consolidated, has grown in importance because of its close relationship to other areas. Thanks to its novelty, little is known regarding its ways of doing scientific research. The recently created Brazilian Journal of Applied Computing (RBCA, in Portuguese) is a representative journal of this focus. Then, in order to have some insight on AC research, the production registered in RBCA has been analyzed. Three analyses have been done: prevalent Scientific Paradigm, Maturity Style and Scientific Approach, over the last 5 years. A checklist has been conceived to help cope with subjectiveness of the Paradigm analysis. All papers were assessed at least two times and most of them three times by different groups. After 501 analyses over three rounds it was found that AC research is, most of all, Technocratic, of beginning Maturity levels and present Development and Descriptive approaches. These features suggest that researchers in AC field have Engineering bias which is reasonable considering the "application" point of view. However, for AC, to become a Computer Science discipline, it seems important that AC researchers use more rigorous scientific methods in order to reach higher levels of credibility.
\end{abstract}

Keywords: Applied computing. Research methodology. Scientific methodology.

\footnotetext{
${ }^{1}$ Programa de Pós-Graduação em Computação Aplicada (PPGCA), Universidade do Estado de Santa Catarina (UDESC) Joinville - Brasil

$\{$ ana.watanabe; marcelo.hounsell\} @udesc.br

\{adelainegelain;marinafouto; rafabosse\} @gmail.com
}

http://dx.doi.org/10.5335/rbca.2014.4182

Revista Brasileira de Computação Aplicada (ISSN 2176-6649), Passo Fundo, v. 6, n. 2, p. 123-135, out. 2014123 


\section{Introdução}

Segundo Denning [1], a ciência aplicada concentra-se no conhecimento de utilidade comprovada. Apesar de não existir uma definição específica e consensual do termo Computação Aplicada, define-se aqui que:

\section{Computação Aplicada (CA) é uma ramificação da área da Ciência da Computação (CC) que usa de metodologias, métodos e técnicas da CC como meio para solucionar problemas, teóricos elou práticos, de outras áreas do conhecimento.}

Esses problemas das outras áreas, por sua vez, podem ser de ordem prática (relacionados ao cotidiano) quanto de ordem teórica (conceitual e fundamental) naquele campo de conhecimento. Semelhante à pesquisa em CC propriamente dita, são usadas técnicas computacionais específicas, mas diferente desta, a preocupação é relacionar a técnica (muitas vezes adaptada) com outras áreas além da própria computação. A multidisciplinaridade e a transdisciplinaridade do trabalho são elementos importantes para a área. Muitas vezes o problema da área de aplicação pode trazer novos desafios para a própria área fundamental da CC, gerando novas técnicas e novos métodos. Portanto, a CA é uma forma diferente de oxigenar a área da CC além de fazer a CC tornar-se mais importante para outras áreas.

A CA já é uma realidade em termos de foco de programas de pós-graduação no Brasil. Alguns desses usam a denominação CA como o Instituto Nacional de Pesquisas Espaciais (Inpe), Universidade Estadual de Feira de Santana (UEFS), Universidade do Rio dos Sinos (UNISINOS), Universidade de Passo Fundo (UPF), Universidade do Estado de Santa Catarina (UDESC), Universidade do Vale do Itajaí (UNIVALI), Universidade de Fortaleza (UNIFOR) e Universidade do Rio Grande do Norte (UFRN), dentre outros.

Cada programa apresenta-se diferente em relação as áreas de CC (áreas de competência) ou área de atuação (área de aplicação). O programa de pós-graduação strictu senso em Computação Aplicada do Inpe tem por objetivo formar pesquisadores com conhecimentos e a competência multidisciplinar necessária em tecnologia da informação, extração de informações, e modelagem computacional, que respondam às necessidades atuais da pesquisa e desenvolvimento em ciências e tecnologias espaciais, em consonância com a missão institucional do Inpe [2]. O programa de pós-graduação em Computação Aplicada da UEFS, concentra-se em pesquisar, desenvolver e aplicar métodos computacionais que auxiliem e viabilizem a solução de problemas das ciências e engenharias, baseando-se na interdisciplinaridade, com a finalidade de promover a criação e a melhoria de algoritmos, hardwares, softwares e sistemas computacionais em geral [3]. Na apresentação do programa da pós-graduação em Computação Aplicada da UDESC vê-se foco em usar a CC nas áreas do Processamento Gráfico, Sistemas Computacionais e Engenharia de Software [4]. O programa de pós-graduação da UPF [5], por sua vez, caracteriza-se por promover a interação entre pesquisa, desenvolvimento e inovação nos segmentos socioeconômicos, em especial, em Tecnologia de Informação, Agricultura e Saúde.

Independente da abordagem e de como são estruturados os programas de pós-graduação na área de CA, são utilizadas metodologias e métodos de pesquisa como toda área de investigação. O que se objetiva neste artigo é analisar como está se desenvolvendo cientificamente a área da CA, olhando para o passado para melhor planejar o futuro. Uma das formas de avaliar, independente do conteúdo específico (área de competência ou área de aplicação), é analisar a estrutura da pesquisa publicada.

Existem vários veículos no Brasil que publicam artigos na área de CA, entretanto, foi selecionada a Revista Brasileira de Computação Aplicada (RBCA). A RBCA é uma publicação da UPF Editora, mantida pelo Programa de Pós-Graduação em Computação Aplicada da UPF, a qual tem por objetivo divulgar a produção científica nacional nas áreas de CA desde a educação, ciências exatas, agricultura e saúde [6]. A RBCA foi selecionada por ser a única que tem Computação Aplicada no nome, o que, certamente, atrai artigos que tenham esse foco; ter sido criada com essa filosofia, e; por ser uma revista ainda nova, essa análise servirá tanto de indícios para a área de CA como um todo, quanto especificamente para os editores e leitores da própria RBCA.

A seleção de um periódico notoriamente com foco exclusivo na CA evita focar em outro periódico (dentre os inúmeros que existem) que contemple CA e CC e ser necessário separar um tipo do outro, o que levaria a uma discussão que, como mencionado, ainda não está consolidada.

Este artigo está organizado da seguinte forma: na seção 2 apresentam-se alguns critérios de verificação da maturidade, paradigmas e tipos de pesquisas; na seção 3 têm-se os trabalhos relacionados à caracterização científica de alguma área; a seção 4 explica a metodologia utilizada para analisar as últimas publicações na RBCA; a seção 5 apresenta uma discussão seguida, na seção 6, das conclusões extraídas dos dados.

Revista Brasileira de Computação Aplicada (ISSN 2176-6649), Passo Fundo, v. 6, n. 2, p. 123-135, out. 2014124 


\section{Classificação da pesquisa científica}

Existem diversas formas para se classificar e analisar o corpo de conhecimento produzido por uma determinada área. Por exemplo, pela linguagem da publicação, temas e tecnologias, entre outros [7]; por categorias definidas pelos autores como teoria, design e modelagem, estudo empírico, validação de hipótese e outros [8-9]; por abordagem ou tipo de pesquisa mais comumente aceitos como formativo, avaliativo, descritivo e desenvolvimento [7] [10], entre outros.

Conforme Wazlawick [11], a diversidade de formas de pesquisa e pouca adesão à metodologia científica acontecem não somente pelo fato de a área da CC ser relativamente nova, mas também pelo fato de que a Computação permeia praticamente todas as atividades humanas e, portanto, inter-relaciona-se com muitas outras disciplinas. É importante que pesquisadores em CC utilizem, de forma adequada, o método científico, para que cada vez mais a CC possa ser respeitada como ciência [11].

Para análise dos artigos foram selecionadas três classificações que têm sido utilizadas para analisar especificamente as subáreas da CC, no caso o paradigma, a maturidade e a abordagem. Ao final, espera-se identificar também qual dessas classificações são mais úteis e interessantes para a área de CA.

\subsection{Paradigmas de pesquisa}

Segundo Eden [12], a CC baseia-se em três paradigmas da pesquisa científica:

- o paradigma racionalista busca obter conhecimento a priori por meio de métodos formais dedutivos, comumente utilizados entre os estudiosos da Teoria da Computação e considera essa área de estudo como um ramo da Matemática;

- o paradigma tecnocrático define a CC como uma disciplina de Engenharia, na qual o conhecimento $a$ posteriori é produzido empiricamente por meio de um conjunto de testes de confiabilidade do programa; preocupa-se em construir sistemas computacionais confiáveis, que resolvam um problema específico, mas sem garantir a generalização da solução proposta;

- o paradigma naturalista define a CC como uma ciência natural (empírica), identifica os conhecimentos $a$ priori e a posteriori em relação ao problema e à sua solução além de combinar a dedução formal e a experimentação científica para validação dos resultados a fim de corroborar ou refutar a hipótese levantada.

A caracterização dos paradigmas proposta por Eden [12] sugere que uma pesquisa possa estar associada a mais de um paradigma, porém, toda pesquisa apresenta um conjunto de características que permitem identificar um paradigma predominante.

\subsection{Maturidade de pesquisa}

De acordo com Wazlawick [11], as produções científicas em CC têm diferentes estilos de maturidade, conforme o grau de conformação dos trabalhos ao método científico e define cinco estilos:

- estilo 1: "apresentação de um produto" é caracterizado pela apresentação de um software como um produto, sem a preocupação com a revisão e com a comparação de trabalhos relacionados nem com a demonstração sobre o quão confiável é o conhecimento produzido.

- estilo 2: "apresentação de algo diferente" consiste em uma forma diferente de resolver um problema, também característicos das áreas emergentes, os trabalhos são apresentados como uma simples comparação entre técnicas, sendo muito mais qualitativas do que quantitativas. Não existe prova com rigor científico e, sim uma tentativa de convencer o leitor do trabalho.

- estilo 3: "apresentação de algo presumidamente melhor" exige que uma nova abordagem apresentada seja comparada quantitativamente com outras abordagens da literatura que seja do estado da arte e demonstra que seu método ou abordagem funciona melhor em determinadas situações, fazendo uso de métricas para mencionar as comparações.

Revista Brasileira de Computação Aplicada (ISSN 2176-6649), Passo Fundo, v. 6, n. 2, p. 123-135, out. 2014125 
- estilo 4: "apresentação de algo reconhecidamente melhor" onde dados empíricos são apresentados em função de testes padronizados e internacionalmente aceitos em um banco de dados conhecido e é relevante para a aceitação dos resultados fazendo uso de métricas aceitas pela comunidade científica de forma que os experimentos possam ser reproduzidos por equipes independentes. Se a nova abordagem for superior às abordagens anteriores, essa passará a ser considerada como o estado da arte.

- estilo 5: “apresentação de uma prova” requer a validação matemática. É necessário apresentar uma teoria e uma prova formal dos seus teoremas.

\subsection{Abordagens de pesquisa}

Dentre as formas de classificação de pesquisas, Morrison e George [10] fornecem uma categorização quanto às abordagens de pesquisa voltada para a área da computação, e destacam quatro tipos de pesquisas geralmente aceitas. São elas:

- formulativa: quando há o desenvolvimento e o refinamento de teorias, modelos ou frameworks que apoiam o progresso científico e governam as atividades de pesquisa na área;

- avaliativa: envolve metodologia que emprega método científico, e, geralmente consiste de teoria ou geração de modelo ou observação seguido pela geração de hipótese e testes;

- descritiva: quando descreve teorias ou modelos para prover o desenvolvimento de unidades da teoria, suas leis de interação, estado do sistema e limites/limitações do modelo. Geralmente, descrevem classes gerais do sistema ou implementações de sistemas específicos, descrevendo suas características, ilustrações da arquitetura e informações de desempenho/usabilidade;

- desenvolvimento: foca no desenvolvimento e na descrição da metodologia e abordagem para suporte ao desenvolvimento de sistemas em geral e desenvolvimento de tipos específicos de sistemas ou componentes de sistemas.

\section{Trabalhos relacionados}

A preocupação em analisar as pesquisas nas subáreas da CC por meio da análise de material publicado em algum veículo científico já foi adotada anteriormente por outros autores.

Claver et al. [13] realizaram uma análise de 1.121 artigos da área de CC publicados nas revistas Information \& Management (IM) e MIS Quarterly (MQ) entre 1981 e 1997. Eles avaliaram os tópicos mais frequentes nas publicações, a metodologia de pesquisa utilizada e os autores que mais publicaram nas revistas analisadas. Quanto à metodologia de pesquisa, classificaram os trabalhos em pesquisas empíricas, as baseadas em estudos de casos e experimentos em campo, com alto rigor de experimentação; e não empíricas, denominadas teóricas, os quais são estudos conceituais e ilustrativos, sem uma validação ou um embasamento formal. Constataram que houve aumento do número de artigos empíricos sobre os teóricos, que ocorreu maior interesse em provar teorias que já existem na prática, indo além da tendência de fazer meras descrições dos fenômenos relacionados ao Sistema de Informação. Demonstraram também, que os autores em sua maioria são norteamericanos e há tendência do agrupamento em equipes de pesquisa, o que consideram indicar um nível mais elevado de maturidade.

A área da CC foi analisada por Wainer et al. [9], os quais observaram as publicações da Association for Computing Machinery (ACM) em 2005. Esses autores constataram uma predominância de $87 \%$ do paradigma tecnocrático e que $26 \%$ dos trabalhos propunham sistemas, modelos ou frameworks, não contemplando nenhum tipo de avaliação. O trabalho mostrou também que houve um aumento no número de trabalhos empíricos, que são fundamentalmente avaliativos de outras contribuições e que reconhecem, portanto, as pesquisas de outros pesquisadores. Entretanto, esse aumento foi pequeno e considerado desencorajador quanto a evolução dos aspectos avaliativos de uma pesquisa na área de CC. Eles finalizam especulando em tons de lamentação que a escassez de trabalhos avaliativos parece se dever ao fato de que a comunidade de CC considera-se criando novas entidades e, por isso, as avaliações empíricas e mais rigorosas estarão sendo consideradas aspectos menos importantes.

Revista Brasileira de Computação Aplicada (ISSN 2176-6649), Passo Fundo, v. 6, n. 2, p. 123-135, out. 2014126 
Magalhães et al. [14] conduziram uma pesquisa para identificar o nível de maturidade dos trabalhos relacionados à Educação a Distância (EAD) publicados no Simpósio Brasileiro de Informática na Educação (SBIE), entre os anos de 2001 e 2009. Para isso, utilizaram como referência a taxonomia proposta por Wazlawick [11], que propõe cinco estilos de maturidade de pesquisa na CC. Nesse contexto, relataram que o estilo mais comum de pesquisa é aquele que tem como resultado a apresentação de uma ferramenta, protótipo, sistema ou novo método para análise de sistemas. Concluíram que a tradição de estilo de pesquisa buscada envolve comumente a criação ou a melhoria de ambientes virtuais de aprendizagem, ferramentas e modelos, sendo a inovação uma característica fundamental nesse processo.

Nesse contexto e por meio dos artigos publicados nos últimos dez anos no Simpósio de Realidade Virtual e Aumentada (SVR) Detroz et al. [7] traçaram um panorama do desenvolvimento da área de Realidade Virtual e Aumentada; complementando o trabalho de Raposo [15], avaliando 262 artigos entre 2004 e 2013. Constataram que artigos sobre desenvolvimento prevaleceram como maioria, no entanto, nos últimos anos, o SVR apresentou maior interesse nas ferramentas de desenvolvimento, técnicas, algoritmos ou metodologias base para suportar o desenvolvimento de aplicações, menos foco nas aplicações e mais nas fundamentações. Os autores salientaram que, em relação à abordagem de pesquisa, devem ser mais incentivadas as pesquisas formulativas, descritivas e avaliativas.

Análises da estrutura da pesquisa em certos nichos (revistas, eventos, áreas) ajudam a entender os seus epistemas e como isso reflete sobre suas práticas e seu futuro. As áreas e subáreas da CC carecem dessas análises, pois são poucos os trabalhos com esse foco, como os já mostrados. Essas análises indicam que a área da CC vem preocupando-se com a evolução quanto às pesquisas publicadas, e mesmo avaliando aspectos diferentes, ajudam a traçar um panorama da área, mostrando suas carências, preferências e tendências. Entretanto, nenhum desses trabalhos fez essa análise no contexto da CA como uma subárea.

\section{Metodologia}

Para obter uma visão dos estudos relacionados à Computação Aplicada no Brasil, foram analisados os artigos da Revista Brasileira de Computação Aplicada (RBCA) dos últimos cinco anos (entre 2009 e 2013), ou seja, desde a criação da revista até o ano passado. O objetivo foi obter uma amostragem dos artigos relacionados a essa área e avaliar que tipo de conhecimento está sendo gerado, a maturidade dos estudos e, por fim, que tipo de solução está sendo obtida.

Essas avaliações foram feitas com base nos trabalhos apresentados em [12] quanto ao paradigma, em [11] quanto ao estilo de maturidade e em [10] quanto a abordagem da pesquisa. Esses critérios foram selecionados pois o paradigma é uma visão geral da CC especificamente para a discussão da área quanto ao viés (ou origem) que esta tem como área afim a matemática, a engenharia ou as ciências naturais. Da mesma forma, os estilos de maturidade foram propostos para classificar os tipos de pesquisa na área de CC como resultado de uma discussão da própria área. Por fim, a abordagem de pesquisa foi utilizada, pois também é uma classificação aplicada à área, mais especificamente proposta para uma subárea (Engenharia de Software), mas que tem sido usada em outras subáreas da CC (ver [15]).

Foram considerados apenas os trabalhos da RBCA classificados como Artigos Originais. Trabalhos dos tipos Relatos de Experiência, Reflexões e Tutoriais não foram abordados. Com isso, dentre os 74 trabalhos publicados na Revista, os 62 artigos originais encontrados foram analisados.

O estudo relacionado à classificação de artigos científicos na área da Computação vem sendo abordado na disciplina de Metodologia da Pesquisa (MEP) do Programa de Pós-Graduação em Computação Aplicada (PPGCA) da Universidade do Estado de Santa Catarina (UDESC) desde 2012. Uma das atividades dessa disciplina foi classificar os artigos da RBCA quanto ao paradigma, maturidade e abordagem. Os resultados apresentados neste trabalho foram compilados a partir das avaliações feitas pelos estudantes da disciplina nos anos de 2012, 2013 e 2014, e novas avaliações foram feitas pelos autores deste artigo apenas em casos de divergência de opiniões entre as avaliações previamente realizadas pelos alunos.

Com isso, no mínimo duas avaliações foram feitas para cada artigo e, em média, três avaliações para cada quesito (paradigma, maturidade e abordagem) de cada artigo foram executadas. Para casos de consenso das opiniões, foi considerada a classificação obtida e, em caso de divergências, o artigo foi analisado novamente, em uma terceira, quarta ou até em uma quinta vez. Além disso, outro critério considerado foi a valorização da 
opinião da turma de 2014, pois nesta houve um maior aprofundamento da discussão em relação aos critérios utilizados nesse estudo: além de ter sido feita uma apresentação das classificações, seguidas de estudo dos artigos respectivos, houve uma análise em outros materiais (trabalhos de conclusão de curso), uma discussão a respeito desta análise, a composição de checklists (as equipes de 2014 propuseram vários modelos) e depois a aplicação das classificações nos artigos da RBCA.

\subsection{Um Checklist para análise do paradigma de pesquisa}

A turma do ano de 2014 da disciplina de MEP foi dividida em equipes de quatro integrantes, que apresentaram como trabalho de disciplina um checklist sobre os paradigmas racionalista, tecnocrático e científico baseado em [12]. A turma de 2013 fez algo semelhante. Portanto, após a análise de quatro propostas de checklists realizados por estas equipes de 2014 e mais uma proposta da turma de 2013, foi montada uma versão do checklist que revisa, estruturada e condensa todas as anteriores, conforme mostrado na Tabela 1.

Tabela 1: Checklist dos paradigmas racionalista, tecnocrático e científico

\begin{tabular}{|c|c|c|}
\hline \multicolumn{3}{|l|}{ Paradigma racionalista } \\
\hline Perguntas & Dimensão & $\begin{array}{l}\text { Marque } \\
\quad \mathrm{X}\end{array}$ \\
\hline $\begin{array}{l}\text { 1) O artigo apresenta similaridade com os ramos da matemática (como lógica, geometria, } \\
\text { álgebra)? }\end{array}$ & Similaridade & \\
\hline 2) O artigo utiliza método de raciocínio lógico ou dedutivo? & Método & \\
\hline $\begin{array}{l}\text { 3) } \mathrm{O} \text { artigo apresenta/trata a correção do programa/artefato de maneira semelhante a } \\
\text { entidades matemáticas? }\end{array}$ & Entidades & \\
\hline 4) O artigo utiliza hipóteses de possíveis relações entre objetos (teoremas/axiomas)? & Procedimentos & \\
\hline 5) $\mathrm{O}$ artigo obtém conhecimento antes (a priori) da execução do programa? & Fonte de conhecimentos & \\
\hline 6) A questão fundamental da pergunta pode ser descrita da forma de "O quê?"? & Pergunta & \\
\hline $\begin{array}{l}\text { 7) } \mathrm{O} \text { artigo efetua avaliação do programa/artefato visando identificar a sua perfeição } \\
\text { (prova)? }\end{array}$ & Avaliação & \\
\hline 8) O conhecimento é aplicado à solução antes de executá-la? & Conhecimento & \\
\hline \multicolumn{3}{|l|}{$\begin{array}{ll}\text { Paradigma tecnocrático } \\
\end{array}$} \\
\hline Perguntas & Dimensão & $\begin{array}{l}\text { Marque } \\
\mathbf{X}\end{array}$ \\
\hline $\begin{array}{l}\text { 1) O artigo apresenta similaridade com a engenharia (como química, elétrica, mecânica, } \\
\text { mecatrônica)? }\end{array}$ & Similaridade & \\
\hline $\begin{array}{l}\text { 2) O artigo utiliza testes de validação (com resultados esperados e conhecidos)? (Se o } \\
\text { teste falhar, o programa/artefato precisa ser revisado). }\end{array}$ & Método & \\
\hline $\begin{array}{l}\text { 3) O artigo apresenta/trata a correção do programa/artefato de maneira semelhante a um } \\
\text { conjunto de dados (caixa preta)? }\end{array}$ & Entidades & \\
\hline $\begin{array}{l}\text { 4) } \mathrm{O} \text { artigo cria declarações formais das funções e interações com o sistema } \\
\text { (especificações)? }\end{array}$ & Procedimentos & \\
\hline $\begin{array}{l}\text { 5) O artigo obtém fonte de conhecimento a posteriori da execução do programa/artefato } \\
\text { (foco no artefato)? }\end{array}$ & Fonte de conhecimentos & \\
\hline 6) A questão fundamental da pergunta pode ser descrita da forma de "Como?"? & Pergunta & \\
\hline $\begin{array}{l}\text { 7) O artigo efetua avaliação do programa/artefato visando identificar à sua adequação } \\
\text { (até onde atende o usuário)? }\end{array}$ & Avaliação & \\
\hline 8) O conhecimento é obtido pelo processo de construção da solução? & Conhecimento & \\
\hline \multicolumn{3}{|l|}{$\begin{array}{ll} & \text { Paradigma científico }\end{array}$} \\
\hline Perguntas & Dimensão & $\begin{array}{l}\text { Marque } \\
\mathbf{X}\end{array}$ \\
\hline $\begin{array}{l}\text { 1) } \mathrm{O} \text { artigo apresenta similaridade com os ramos da ciência natural (como astronomia, a } \\
\text { biologia e medicina)? }\end{array}$ & Similaridade & \\
\hline $\begin{array}{l}\text { 2) } \mathrm{O} \text { artigo utiliza experimentos e coleta de dados (com resultados que precisam ser } \\
\text { tratados)? (Se o experimento falhar, a teoria precisa ser revista). }\end{array}$ & Método & \\
\hline $\begin{array}{l}\text { 3) O artigo apresenta/trata a correção do programa/artefato de maneira semelhante a } \\
\text { processos mentais (consegue prever comportamentos)? }\end{array}$ & Entidades & \\
\hline 4) O artigo observa uma possível recorrência ou padrão de fenômenos (hipóteses)? & Procedimentos & \\
\hline $\begin{array}{l}\text { 5) O artigo obtém fonte de conhecimento a posteriori da execução do programa/artefato } \\
\text { (foco no comportamento)? }\end{array}$ & Fonte de conhecimentos & \\
\hline 6) A questão fundamental da pergunta é na forma de "Por quê?"? & Pergunta & \\
\hline $\begin{array}{l}\text { 7) O artigo efetua avaliação do programa/artefato visando identificar a sua pertinência } \\
\text { (se aplica ou não a uma situação ou a um comportamento)? }\end{array}$ & Avaliação & \\
\hline 8) O conhecimento é obtido pelos resultados da aplicação da solução. & Conhecimento & \\
\hline
\end{tabular}


O checklist da Tabela 1 apresenta, para cada paradigma, oito perguntas vinculadas a uma determinada dimensão comum a todos os paradigmas.

- a dimensão similaridade (pergunta n. 1) busca identificar qual ramo o artigo tem maior similaridade (Matemática, Engenharia ou Ciência Natural);

- a dimensão método (pergunta n. 2) verifica qual o método utilizado pelo artigo (raciocínio lógico, teste de validação ou experimentos e coletas de dados);

- a dimensão entidades (pergunta n. 3) verifica como o artigo apresenta ou trata a correção do programa ou do artefato (entidades matemáticas, conjunto de dados ou processos mentais);

- a dimensão procedimentos (pergunta n. 4) busca saber qual o procedimento adotado pelo artigo (teoremas/axiomas, especificações ou hipóteses);

- a dimensão fonte de conhecimentos (pergunta n. 5) tenta identificar se o artigo obtém conhecimento antes (a priori) ou depois (a posteriori), verificando se o foco é no artefato ou no comportamento;

- a dimensão pergunta (pergunta n. 6) busca identificar qual a questão fundamental que o artigo visa responder (o quê, como ou por que);

- a dimensão avaliação (pergunta n. 7) busca saber como o artigo efetua avaliação do programa ou artefato (prova, se atende ao usuário ou a uma situação/comportamento);

- a dimensão conhecimento (pergunta n. 8) visa saber como o conhecimento é adquirido no artigo (antes de executar, no processo ou pelo resultado).

Assim, para verificar qual o paradigma dominante de um determinado artigo deve-se, após ler o artigo, marcar com $\mathrm{X}$ os itens que mais se adequaram ao conteúdo de cada paradigma. Depois, deve-se contar o que mais obteve pontuações para concluir qual o paradigma dominante do referido artigo. Se ocorrer empate, deve-se reavaliar cada pergunta, observando que nem todas as perguntas são mutuamente exclusivas, o que sugere que um artigo possa apresentar componentes de mais de um paradigma. Lembrando que o objetivo do checklist é justamente ajudar a identificar o paradigma que é dominante.

\section{Dados}

Durante o desenvolvimento dos trabalhos na disciplina de MEP, cada equipe elaborou uma forma diferente de classificar os artigos, algumas usaram checklist, mas outras não. Devido às particularidades na avaliação de cada equipe, algumas divergências foram encontradas.

Na Tabela 2 apresenta-se a relação dos artigos analisados e identificados pela edição (volume, número e página do artigo); as colunas $\mathrm{P}, \mathrm{E}$ e A indicam a concordância obtida após a primeira rodada de avaliação respectivamente para o critério do paradigma, estilo e abordagem. As cores indicam vermelho quando não houve consenso inicial, amarelo quando houve uma maioria e verde quando houve consenso. As três últimas colunas indicam o resultado final das avaliações para cada artigo e cada critério. Os respectivos artigos da RBCA estão representados na Tabela 2, nas primeiras colunas, pelo volume, número da edição e páginas do artigo.

No link https://dl.dropboxusercontent.com/u/24168143/AvaliacoesArtigosRBCA.xlsx do Dropbox, está disponível a planilha completa, contendo o resultado das avaliações de todas as rodadas. Nesta versão, é possível verificar a evolução das avaliações parciais, até que se conseguisse chegar a uma conclusão sobre como classificar o artigo. A planilha do link foi organizada da seguinte forma:

1. Informações sobre o artigo - edição, autores e páginas da revista.

2. Primeira rodada $-1^{\mathrm{a}}, 2^{\mathrm{a}}$ e $3^{\mathrm{a}}$ avaliações:

a. em cores tem-se as primeiras avaliações: $1^{\mathrm{a}}, 2^{\mathrm{a}}$ e $3^{\mathrm{a}}$ referentes aos trabalhos realizados pelos alunos da disciplina de MEP das turmas de 2014, 2013 e 2012;

b. coluna Merge das primeiras avaliações: havendo consenso de pelo menos duas opiniões (sendo uma delas da turma 2014 - como já foi explicado), é considerado o conceito. Caso os três conceitos sejam diferentes, o artigo será reavaliado;

c. visão parcial do resultado por meio de cores. 
3. Segunda rodada $-4^{\mathrm{a}}$ avaliação:

a. $\quad 4^{a}$ avaliação: apenas artigos que apresentaram conflitos nas primeiras avaliações, indicados em vermelho, foram reavaliados pelos autores deste trabalho;

b. Merge da $4^{a}$ avaliação com as primeiras avaliações: é considerado o consenso que tiver duas opiniões iguais, sendo uma delas a $4^{\mathrm{a}}$ avaliação. Caso os resultados ainda tenham divergência, o artigo será reavaliado;

c. Visão parcial do resultado por intermédio de cores, sendo que os artigos que não tiveram consenso continuam em vermelho, já os que tiverem, são apresentados em amarelo.

4. Terceira rodada $-5^{\mathrm{a}}$ avaliação:

a. $5^{\text {a }}$ avaliação: apenas os artigos que continuam com divergência foram avaliados pelo autor deste artigo com maior experiência;

b. Merge da $5^{\mathrm{a}}$ avaliação com os resultados anteriores, os quais continuam sendo considerados o consenso quando existirem duas opiniões iguais, tendo maior peso avaliações mais recentes:

c. Visão do resultado por meio de cores.

5. Número de avaliações:

a. Indica o número de avaliações feitas para cada critério, para cada artigo.

A maioria das divergências observadas foi nos quesitos estilo e abordagem. Observou-se que nas avaliações das turmas de 2013 e de 2012, muitas vezes, o artigo analisado utilizava algumas palavras no decorrer do texto que tinham relação com algo que descrevesse um estilo ou abordagem, como por exemplo, utilização da palavra avaliação no meio do texto fazia com que o artigo fosse classificado como pesquisa de abordagem avaliativa, o que não era necessariamente verdadeiro no âmbito geral do trabalho. Isso ocorreu em várias análises e, portanto, muita atenção foi necessária para que uma classificação mais condizente com o tipo do artigo fosse obtida sem ser influenciada pelo uso de palavras relacionadas no texto.

O esquema de cores utilizado na análise dos artigos foi determinado de acordo com a existência ou não de consenso entre as avaliações. Na primeira avaliação feita, analisando os resultados obtidos pelos trabalhos das equipes durante os anos de 2012 a 2014:

- quando todas as opiniões eram iguais, foi atribuída à avaliação a cor verde, indicando que aquela análise obteve um resultado final, na qual todos concordaram com o parâmetro avaliado para determinado trabalho;

- a cor amarela foi atribuída quando existia consenso parcial entre as opiniões, ou seja, havia maioria, mas essa não era absoluta;

- quando as opiniões avaliadas fossem todas divergentes, a cor atribuída para a classificação foi a vermelha, indicando que não houve consenso nenhum entre os grupos em relação ao conceito avaliado. Nesses casos, os artigos passaram por novas avaliações até que fosse atingido consenso parcial e a cor vermelha passasse a ser amarela para todos os casos analisados;

- a cor branca foi usada nas situações em que não se tinha informações suficientes para decidir inicialmente.

Vale ressaltar que o artigo v1n1p30, segunda coluna da Tabela 2 está em branco, pois não é um trabalho de pesquisa primária e sim um levantamento bibliográfico e, por conseguinte, as classificações apresentadas não se aplicam a esse tipo de artigo. Com isso, dos 62 artigos originais, as análises são todas referentes a 61 desses.

Das colunas dos números de avaliações executadas para cada classificação obtém-se que a média de avaliações de paradigmas foi de 2,92 avaliações, sendo a moda 3, de estilos de maturidade foi 2,90 sendo a moda 3 e, de abordagens foi 2,23 sendo a moda 2. Somando um total de 501 avaliações para os 61 artigos da RBCA.

A Tabela 3 mostra, em números percentuais, situações de consenso em cada rodada. E na Figura 2 é mostrado um gráfico com a evolução da situação das classificações, em números absolutos, a cada rodada de análise: são três resultados para cada classificação apresentada no artigo, onde P: Paradigma, E: Estilo e A: Abordagem, referem-se respectivamente a:

- após a primeira rodada de avaliações, com a situação com base nos dados acumulados das turmas dos anos de 2014, 2013 e 2012;

- após a segunda rodada de reavaliação (com a inserção de uma $4^{a}$ avaliação em muitos casos); 
- ao final da terceira e última rodada de avaliações (em alguns casos com a inserção de uma $5^{\text {a }}$ avaliação).

Tabela 2: Relação das divergências na compilação dos dados

\begin{tabular}{|c|c|c|c|c|c|c|c|c|c|c|}
\hline \multirow{2}{*}{ Edição } & \multirow[b]{2}{*}{ Páginas } & \multicolumn{3}{|c|}{ Avaliação final } & \multicolumn{3}{|c|}{ Consenso } & \multicolumn{3}{|c|}{ N. avaliações } \\
\hline & & Paradigma & \begin{tabular}{|l|} 
Estilo \\
\end{tabular} & Abordagem & $\mathrm{P}$ & $\mathrm{E}$ & $\mathrm{A}$ & Nr. P & Nr. E & Nr. A \\
\hline v. 1, n. 1 (2009) & $15-29$ & 1-Tecnocrata & Estilo 2 & 4-Desenvolvimento & 1 & 3 & 1 & 3 & 4 & 2 \\
\hline v. 1 , n. 1 (2009) & $30-41$ & - & - & - & 0 & 0 & 0 & 2 & 2 & 1 \\
\hline v. 1 , n. 1 (2009) & $42-52$ & 1-Tecnocrata & Estilo 1 & 3-Descritiva & 3 & 3 & 3 & 4 & 3 & 2 \\
\hline v. 1 , n. 1 (2009) & $53-64$ & 3-Científico & Estilo 3 & 4-Desenvolvimento & 2 & 3 & 1 & 3 & 4 & 2 \\
\hline v. 1 , n. 1 (2009) & $65-76$ & 1-Tecnocrata & Estilo 2 & 4-Desenvolvimento & 1 & 2 & 1 & 3 & 3 & 2 \\
\hline v. 1 , n. 1 (2009) & $77-91$ & 1-Tecnocrata & Estilo 1 & 4-Desenvolvimento & 1 & 1 & 1 & 3 & 3 & 2 \\
\hline v. 2, n. $1(2010)$ & $02-16$ & 1-Tecnocrata & Estilo 1 & 4-Desenvolvimento & 2 & 3 & 3 & 3 & 4 & 3 \\
\hline v. 2 , n. $1(2010)$ & $17-27$ & 1-Tecnocrata & Estilo 1 & 4-Desenvolvimento & 1 & 1 & 1 & 3 & 3 & 2 \\
\hline v. 2 , n. 1 (2010) & $28-40$ & 1-Tecnocrata & Estilo 1 & 4-Desenvolvimento & 2 & 2 & 3 & 3 & 3 & 3 \\
\hline v. 2 , n. 1 (2010) & $41-56$ & 1-Tecnocrata & Estilo 2 & 3-Descritiva & 2 & 3 & 3 & 3 & 4 & 3 \\
\hline v. 2 , n. $1(2010)$ & $57-68$ & 1-Tecnocrata & Estilo 1 & 3-Descritiva & 2 & 1 & 1 & 3 & 3 & 2 \\
\hline v. 2 , n. $1(2010)$ & $69-78$ & 1-Tecnocrata & Estilo 1 & 4-Desenvolvimento & 1 & 2 & 1 & 3 & 3 & 2 \\
\hline v. 2 , n. $1(2010)$ & $79-88$ & 1-Tecnocrata & Estilo 2 & 4-Desenvolvimento & 2 & 2 & 1 & 3 & 3 & 2 \\
\hline v. 2 , n. $1(2010)$ & $89-99$ & 1-Tecnocrata & Estilo 2 & 3-Descritiva & 1 & 3 & 1 & 3 & 4 & 2 \\
\hline v. 2 , n. 2 (2010) & $58-68$ & 1-Tecnocrata & Estilo 1 & 3-Descritiva & 1 & 1 & 1 & 3 & 3 & 2 \\
\hline v. 2, n. $2(2010)$ & $69-80$ & 1-Tecnocrata & Estilo 2 & 4-Desenvolvimento & 1 & 3 & 1 & 3 & 4 & 2 \\
\hline v. 2 , n. $2(2010)$ & $81-95$ & 1-Tecnocrata & Estilo 1 & 3-Descritiva & 1 & 2 & 1 & 3 & 3 & 2 \\
\hline v. 3, n. 1 (2011) & $43-52$ & 1-Tecnocrata & Estilo 1 & 4-Desenvolvimento & 1 & 2 & 1 & 3 & 3 & 2 \\
\hline v. 3, n. 1 (2011) & $53-63$ & 1-Tecnocrata & Estilo 1 & 4-Desenvolvimento & 1 & 1 & 1 & 3 & 3 & 2 \\
\hline v. 3 , n. 1 (2011) & $64-73$ & 1-Tecnocrata & Estilo 1 & 4-Desenvolvimento & 1 & 2 & 1 & 3 & 3 & 2 \\
\hline v. 3 , n. 1 (2011) & $74-81$ & 3-Científico & Estilo 3 & 2-Avaliativa & 2 & 2 & 1 & 3 & 3 & 2 \\
\hline v. 3 , n. $1(2011)$ & $82-91$ & 1-Tecnocrata & Estilo 2 & 4-Desenvolvimento & 1 & 1 & 1 & 3 & 3 & 2 \\
\hline v. 3, n. 2(2011) & $17-29$ & 1-Tecnocrata & Estilo 1 & 4-Desenvolvimento & 3 & 3 & 1 & 4 & 4 & 2 \\
\hline v. 3 , n. 2 (2011) & $30-45$ & 1-Tecnocrata & Estilo 1 & 4-Desenvolvimento & 1 & 2 & 1 & 3 & 3 & 2 \\
\hline v. 3 , n. 2 (2011) & $46-57$ & 1-Tecnocrata & Estilo 1 & 4-Desenvolvimento & 1 & 1 & 1 & 3 & 3 & 2 \\
\hline v. 3 , n. 2 (2011) & $58-72$ & 1-Tecnocrata & Estilo 2 & 4-Desenvolvimento & 1 & 2 & 1 & 3 & 3 & 2 \\
\hline v. 3 , n. 2 (2011) & $73-90$ & 1-Tecnocrata & Estilo 1 & 4-Desenvolvimento & 1 & 2 & 1 & 3 & 3 & 2 \\
\hline v. 3 , n. 2 (2011) & $91-102$ & 2-Racionalista & Estilo 3 & 1-Formulativa & 2 & 2 & 1 & 3 & 3 & 2 \\
\hline v. 3 , n. $2(2011)$ & $103-117$ & 1-Tecnocrata & Estilo 1 & 4-Desenvolvimento & 1 & 2 & 1 & 3 & 3 & 2 \\
\hline v. 4, n. 1 (2012) & $12-24$ & 1-Tecnocrata & Estilo 1 & 4-Desenvolvimento & 1 & 1 & 3 & 3 & 3 & 3 \\
\hline v. 4 , n. 1 (2012) & $25-37$ & 1-Tecnocrata & Estilo 2 & 4-Desenvolvimento & 1 & 3 & 3 & 3 & 4 & 3 \\
\hline v. 4 , n. 1 (2012) & $38-47$ & 1-Tecnocrata & Estilo 2 & 3-Descritiva & 2 & 3 & 3 & 3 & 3 & 3 \\
\hline v. 4 , n. 1 (2012) & $48-60$ & 1-Tecnocrata & Estilo 1 & 3-Descritiva & 1 & 1 & 1 & 3 & 3 & 2 \\
\hline v. 4 , n. 1 (2012) & $61-70$ & 2-Racionalista & Estilo 3 & 4-Desenvolvimento & 3 & 3 & 1 & 4 & 3 & 2 \\
\hline v. 4 , n. 1 (2012) & $71-80$ & 1-Tecnocrata & Estilo 3 & 2-Avaliativa & 2 & 2 & 1 & 3 & 3 & 2 \\
\hline v. 4 , n. 1 (2012) & $81-91$ & 3-Científico & Estilo 1 & 2-Avaliativa & 2 & 2 & 1 & 3 & 3 & 2 \\
\hline v. 4 , n. 1 (2012) & $92-101$ & 1-Tecnocrata & Estilo 1 & 3-Descritiva & 1 & 1 & 1 & 3 & 3 & 2 \\
\hline v. 4 , n. 1 (2012) & $102-111$ & 1-Tecnocrata & Estilo 1 & 3-Descritiva & 1 & 2 & 3 & 3 & 3 & 3 \\
\hline v. 4 , n. 2 (2012) & $2-15$ & 1-Tecnocrata & Estilo 1 & 4-Desenvolvimento & 1 & 1 & 3 & 2 & 2 & 3 \\
\hline v. 4 , n. 2 (2012) & $16-27$ & 1-Tecnocrata & Estilo 1 & 4-Desenvolvimento & 1 & 1 & 1 & 2 & 2 & 2 \\
\hline v. 4 , n. 2 (2012) & $28-41$ & 1-Tecnocrata & Estilo 1 & 3-Descritiva & 1 & 1 & 1 & 2 & 2 & 2 \\
\hline v. 4 , n. $2(2012)$ & $42-57$ & 3-Científico & Estilo 4 & 2-Avaliativa & 1 & 0 & 1 & 2 & 2 & 2 \\
\hline v. 4 , n. 2 (2012) & $58-71$ & 1-Tecnocrata & Estilo 1 & 3-Descritiva & 3 & 3 & 1 & 3 & 3 & 2 \\
\hline v. 4 , n. 2 (2012) & $72-82$ & 3-Científico & Estilo 3 & 2-Avaliativa & 1 & 0 & 1 & 2 & 2 & 2 \\
\hline v. 4 , n. 2 (2012) & $83-91$ & 1-Tecnocrata & Estilo 1 & 4-Desenvolvimento & 1 & 1 & 3 & 2 & 2 & 3 \\
\hline v. 4 , n. 2 (2012) & $92-102$ & 1-Tecnocrata & Estilo 1 & 4-Desenvolvimento & 3 & 1 & 1 & 3 & 2 & 2 \\
\hline v. 5, n. $1(2013)$ & $2-13$ & 1-Tecnocrata & Estilo 3 & 3-Descritiva & 2 & 2 & 3 & 3 & 3 & 3 \\
\hline v. 5 , n. 1 (2013) & $14-25$ & 1-Tecnocrata & Estilo 2 & 2-Avaliativa & 1 & 2 & 3 & 3 & 3 & 3 \\
\hline v. 5 , n. 1 (2013) & $26-41$ & 1-Tecnocrata & Estilo 1 & 3-Descritiva & 1 & 2 & 1 & 3 & 3 & 2 \\
\hline v. 5, n. 1 (2013) & $42-54$ & 1-Tecnocrata & Estilo 3 & 2-Avaliativa & 1 & 2 & 3 & 3 & 3 & 3 \\
\hline v. 5, n. 1 (2013) & $55-70$ & 1-Tecnocrata & Estilo 1 & 3-Descritiva & 1 & 2 & 1 & 3 & 3 & 2 \\
\hline v. 5 , n. 1 (2013) & $71-83$ & 1-Tecnocrata & Estilo 1 & 3-Descritiva & 1 & 2 & 3 & 3 & 3 & 3 \\
\hline v. 5 , n. 1 (2013) & $84-97$ & 1-Tecnocrata & Estilo 1 & 3-Descritiva & 1 & 2 & 3 & 3 & 3 & 3 \\
\hline v. 5 , n. 1 (2013) & $98-112$ & 1-Tecnocrata & Estilo 1 & 2-Avaliativa & 3 & 2 & 1 & 4 & 3 & 2 \\
\hline v. 5, n. $1(2013)$ & $113-125$ & 3-Científico & Estilo 3 & 2-Avaliativa & 3 & 2 & 1 & 4 & 3 & 2 \\
\hline v. 5, n. 2 (2013) & $28-39$ & 1-Tecnocrata & Estilo 1 & 2-Avaliativa & 1 & 2 & 3 & 2 & 2 & 3 \\
\hline v. 5 , n. 2 (2013) & $40-54$ & 1-Tecnocrata & Estilo 3 & 4-Desenvolvimento & 1 & 3 & 1 & 2 & 4 & 2 \\
\hline v. 5 , n. 2 (2013) & $55-68$ & 1-Tecnocrata & Estilo 1 & 4-Desenvolvimento & 1 & 2 & 1 & 2 & 2 & 2 \\
\hline v. 5 , n. 2 (2013) & $69-84$ & 1-Tecnocrata & Estilo 2 & 3-Descritiva & 1 & 2 & 0 & 2 & 2 & 2 \\
\hline v. 5 , n. 2 (2013) & $85-95$ & 1-Tecnocrata & Estilo 2 & 2-Avaliativa & 3 & 2 & 0 & 4 & 2 & 2 \\
\hline v. 5 , n. 2 (2013) & 96-109 & 1-Tecnocrata & Estilo 2 & 2-Avaliativa & 1 & 2 & 0 & 2 & 2 & 2 \\
\hline v. 5 , n. 2 (2013) & 110-119 & 1-Tecnocrata & Estilo 2 & 3-Descritiva & 3 & 3 & 0 & 4 & 3 & 2 \\
\hline
\end{tabular}

Os valores percentuais resultantes das 501 análises, em três rodadas de avaliação, dos 61 artigos publicados na RBCA estão mostrados de maneira resumida nos gráficos da Figura 1. 
A Figura 1 mostra que o paradigma mais presente na área de CA é o tecnocrata, respondendo com $87 \%$ dos artigos avaliados ao passo que o menos frequente é o paradigma racionalista, respondendo por apenas $3 \%$ dos artigos. Dentre os estilos de maturidade, encontrou-se 57\% de artigos do estilo 1 de maturidade apresentação de produto; e $25 \%$ do estilo 2 de maturidade - apresentação de algo diferente.

Figura 1: Gráfico dos percentuais das análises

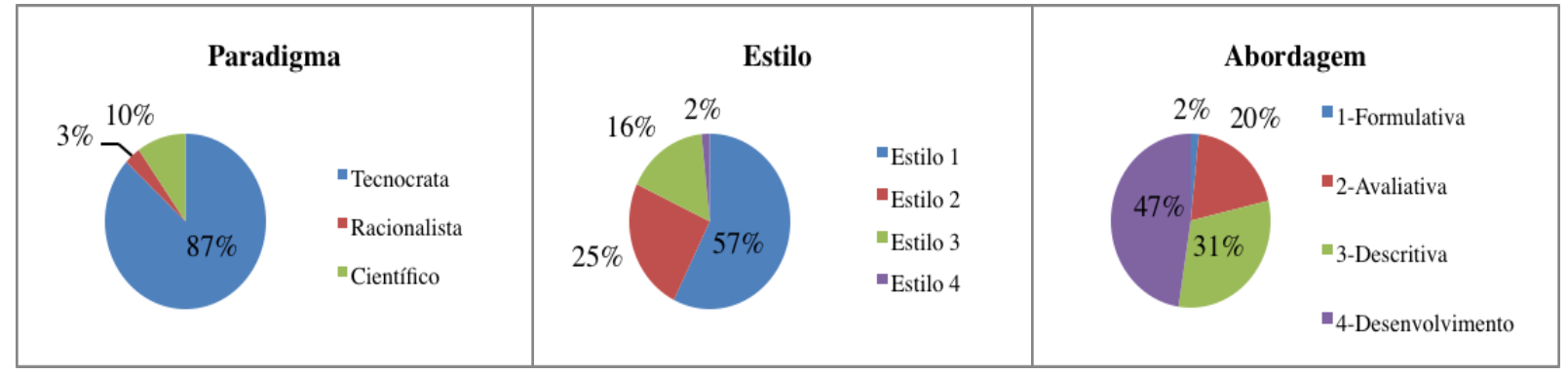

Apenas um único artigo foi classificado como do estilo 4 de maturidade - apresentação de algo comprovadamente melhor. Este artigo foi classificado como tendo abordagem formativa e paradigma racionalista. Quanto à abordagem, 47\% dos artigos apresentavam abordagem desenvolvimentista e apenas $2 \%$ foram caracterizados como formulativa.

Tabela 3: Tabulação dos consensos a cada rodada

\begin{tabular}{|c|c|c|c|c|}
\hline Avaliações & & Vermelho & Amarelo & Verde \\
\hline $1^{\text {a }}$ Rodada & $\mathrm{P}$ & $15 \%$ & $20 \%$ & $\mathbf{6 6 \%}$ \\
\hline & $\mathrm{E}$ & $23 \%$ & $49 \%$ & $25 \%$ \\
\hline & $\mathrm{A}$ & $26 \%$ & $0 \%$ & $\mathbf{6 7 \%}$ \\
\hline $2^{\mathrm{a}}$ Rodada & $\mathrm{P}$ & $5 \%$ & $30 \%$ & $66 \%$ \\
\hline & $\mathrm{E}$ & $11 \%$ & $64 \%$ & $25 \%$ \\
\hline & $\mathrm{A}$ & $26 \%$ & $0 \%$ & $67 \%$ \\
\hline $3^{\mathrm{a}}$ Rodada & $\mathrm{P}$ & $0 \%$ & $34 \%$ & $66 \%$ \\
\hline & $\mathrm{E}$ & $0 \%$ & $74 \%$ & $25 \%$ \\
\hline
\end{tabular}

Observe na Tabela 3 e na Figura 2 que os consensos iniciais (colunas verdes) não se modificaram ao longo das reavaliações, pois remetiam-se somente aos casos de consenso total; que as rodadas de avaliação concorreram para diminuir as situações de completa falta de consenso (colunas vermelhas) e, consequentemente, o aumento dos consensos parciais (colunas amarelas).

Se forem tomados somente os 53 artigos do paradigma tecnocrata, observa-se que $34(64 \%)$ desses apresenta estilo de maturidade 1 e, $15(38 \%)$ do estilo 2. Desses, 27 (51\%) usaram abordagem desenvolvimentista e 19 (36\%) descritiva.

\section{Discussão}

Da Tabela 3 observa-se um elevado percentual de definição obtido já na primeira rodada, para a análise do Paradigma e da Abordagem (66\% e 67\%, respectivamente), que indicam que esses critérios não se mostraram difíceis de serem avaliados pelas várias turmas. Todos os artigos foram avaliados pelo menos duas vezes, pois a moda para a maturidade e abordagem foi 2 e para paradigma 3, sendo pelo menos uma das avaliações a da turma de 2014. Para essa avaliação foi dada uma importância maior, pois essa turma fez um estudo mais aprofundado nos critérios adotados pela pesquisa. A maioria dos artigos passou por três avaliações quanto ao paradigma e, em todos os casos, quando persistiu discordância, um coautor com maior experiência executou analises finais. 
Parece haver uma correlação forte entre o paradigma tecnocrático e os estilos de maturidade 1 e 2, assim como para com as abordagens desenvolvimentista e descritiva, uma vez que os percentuais relativos desses dois últimos critérios foram acentuados quando se avaliou somente os artigos tecnocráticos. Entretanto, a literatura não indica isso como uma restrição e, com efeito, artigos de Estilos mais maduros, assim como abordagens avaliativas foram encontrados dentro do próprio conjunto de análise. Logo, vê-se que é possível, mesmo adotando paradigma Tecnocrático, efetuar pesquisa científica mais rigorosa em estilo de Maturidade superior com abordagem mais avaliativa.

Figura 2: Gráfico evolução classificações

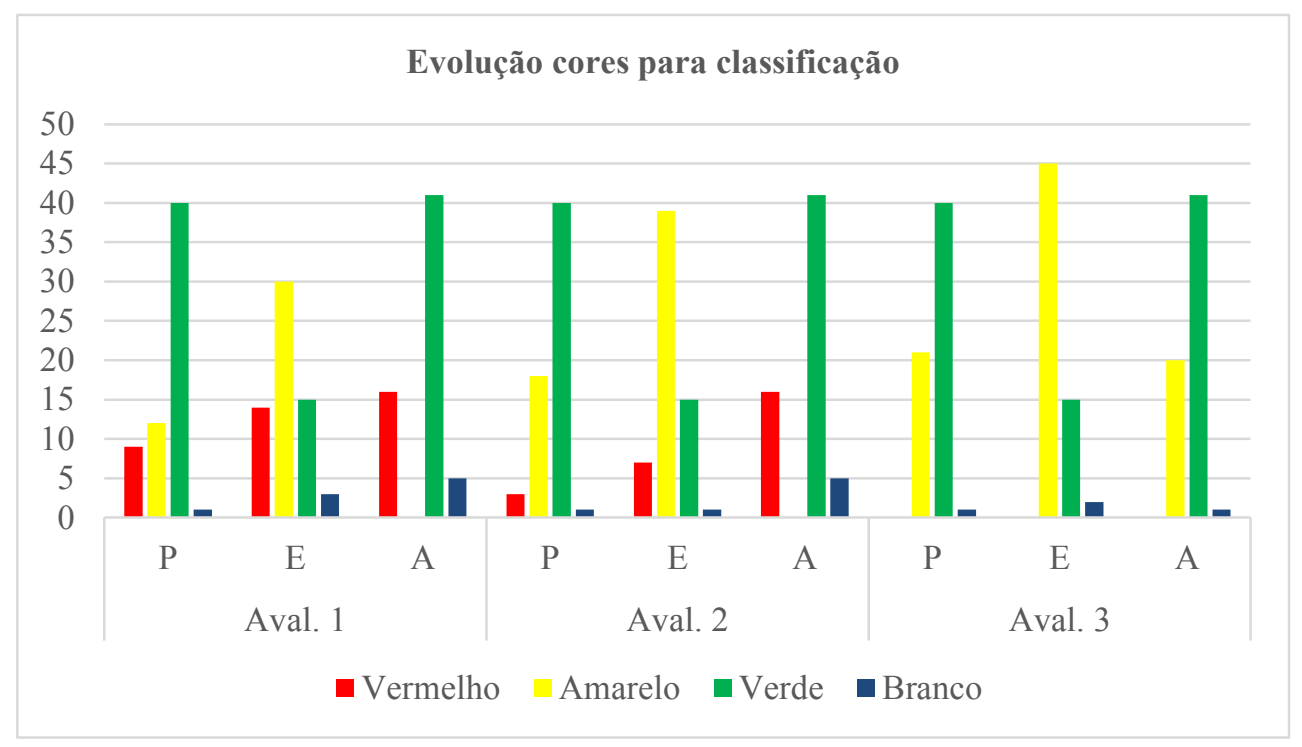

A utilização do checklist ajudou a analisar o paradigma científico dos artigos a ponto de padronizar e minimizar discrepâncias de avaliações. Indicativo da utilidade do checklist é o fato de que as discrepâncias graves caíram de $15 \%$ para $5 \%$, da $1^{\mathrm{a}}$ para a $2^{\mathrm{a}}$ rodada de avaliações, conforme Tabela 3.

A análise dos estilos de maturidade, apesar de serem compostos por elementos cumulativos aparentemente de fácil identificação, provou-se menos trivial de ser executada. Indicativo dessa dificuldade é o fato de que apenas $25 \%$ dos artigos avaliados sob esse critério apresentaram consenso na $1^{\text {a }}$ rodada de avaliação (Tabela 3). Isso deveu-se a necessidade de qualificar se as eventuais (apesar de poucas) avaliações feitas nos artigos utilizavam modelos comparativos difundidos na comunidade cientifica (base de testes relevantes) com métricas também reconhecidas - o que requereu grande esforço, pois os artigos cobriam uma ampla variedade de subáreas da Ciência da Computação. Já a abordagem de pesquisa identifica, sobretudo, a ênfase da pesquisa, apesar desta poder contemplar outros elementos do processo científico - o que tornou a separação entre os tipos não tão trivial também.

Analisando cada uma das classificações, vê-se utilidade para todas essas, pois ressaltam aspectos diferentes, tais como: o viés científico, os elementos da pesquisa e os objetivos gerais. Entretanto, os dois primeiros foram concebidos para a área da $\mathrm{CC}$ como um todo e, portanto, pelo processo lógico dedutivo, passíveis de encaixarem-se em CA. Entretanto, o último foi concebido para a subárea da Engenharia de Software e, portanto, pelo processo lógico indutivo, sua adequação à área de CA, que supostamente seria mais abrangente, precisa ser melhor avaliada.

Pelos dados obtidos, pode-se observar que semelhante ao que foi encontrado em estudos anteriores [5], [7], [9] a CA segue fundamentalmente a epistemologia das Engenharias. Entretanto, semelhante ao encontrado em [9], surpreende o número de trabalhos com Estilo de Maturidade 1 (57\%), mas também Estilo 2 (com 25\%), onde praticamente não há preocupação em executar uma avaliação mais rigorosa dos artefatos resultantes das pesquisas, sejam esses softwares, hardwares ou até modelos. Ou seja, em índices muito superiores (mais que o dobro dos $26 \%$ encontrados em [5]) ao que apresentavam os artigos publicados por uma entidade de renome internacional, há quase uma década atrás, para a área de CC como um todo. 


\section{Conclusão}

A área da Computação Aplicada (CA), uma ramificação da Ciência da Computação (CC), com preocupação em usar métodos e técnicas da CC para solucionar problemas, teóricos ou práticos, de outras áreas do conhecimento, é uma ênfase recente, mas já presente em alguns programas de pós-graduação do Brasil e já dispondo de um veículo de publicação específico, a Revista Brasileira de Computação Aplicada (RBCA). Como forma de se avaliar como caracterizam-se as pesquisas em CA, tomou-se como amostra as publicações da RBCA entre 2009 e 2013

Utilizou-se como critério de avaliação classificações relacionadas à área de CC com foco no Paradigma Científico, na Maturidade da Pesquisa e na Abordagem de Pesquisa. Após 501 análises feitas em três rodadas distintas, envolvendo mestrandos de três turmas, de três anos consecutivos, avaliou-se os 61 artigos primários publicados na RBCA. Como a aplicação do critério do paradigma mostrava-se a mais subjetiva e abrangente (somente três itens de classificação), um checklist foi concebido pela convergência da análise de cinco propostas iniciais. Esse checklist revelou-se útil para facilitar a avaliação desse critério. Nenhuma das avaliações mostrouse trivial apesar de definições iniciais logo no início para os critérios de Paradigma e Abordagem, mas estes podem ter sido influenciados pela concentração de artigos em poucos tipos desses critérios.

O paradigma Tecnocrático apareceu em 87\%, o Estilo 1 de Maturidade em $57 \%$ e a Abordagem Desenvolvimentista apareceu em $47 \%$ dos artigos. Os dados obtidos revelaram-se coerentes e compatíveis com a caracterização da grande área de $\mathrm{CC}$, conforme encontrado em trabalhos relacionados. Considerando os aspectos epistemológicos de cada classificação, vê-se coerência na tríade encontrada: tecnocrática-baixa maturidadedesenvolvimentista, que de modo simplificado, acaba por caracterizar os artigos da área de Computação Aplicada.

Apesar de coerente, vê-se com preocupação uma predominância tão acentuada, principalmente quanto aos Estilos de Maturidade, pois para manter-se um elevado padrão científico espera-se que sejam feitas avaliações rigorosas das soluções encontradas, o que não tem ocorrido. A limitada utilização de avaliações das propostas concorre também para limitar o impacto dos trabalhos, pois causa incertezas quanto à sua efetividade e real utilidade, limitando também a difusão da própria solução proposta. Assim, em benefício de uma maior difusão das soluções de integração da CC com outras áreas, vê-se como benéfico que a comunidade de CA adote posturas mais avaliativas de seus trabalhos, sem, entretanto, abdicar dos aspectos de inovação característicos da área.

Reconhece-se a limitação desta pesquisa em relação à área de CA como um todo pelo próprio fato de a RBCA ser uma das várias revistas que publicam material de CA, de ser uma revista recente e pelo fato de que tem foco nas áreas de aplicação de educação, ciências exatas, agricultura e saúde. Entretanto, espera-se que esse levantamento sirva de motivo de reflexão para eventos e revistas da área de CA, assim como o foi para a área de CC ocorrido anteriormente com o intuito de que a área se consolide e, a área da Computação, como um todo, torne-se cada vez mais Ciência.

A ampliação desse estudo para outros periódicos que contemplem outras subáreas faz-se necessária para complementar e comparar com os dados apresentados aqui. Recomenda-se ainda, em função do esforço depreendido na classificação dos Estilos de Maturidade e da Abordagem de Pesquisa, e como forma de também padronizá-los e sistematizá-los, a confecção de checklists para esses critérios com o intuito de facilitar e enfatizar os aspectos a serem discriminados. Ainda, aplicar levantamento semelhante a este para artigos mais recentes de outros veículos de publicação notoriamente mais impactantes poderia ajudar a indicar quais os melhores Paradigmas, Estilos de Maturidade e/ou Abordagens a serem almejados.

\section{Agradecimentos}

Os autores gostariam de agradecer a todos os acadêmicos das turmas dos anos de 2012, 2013 e 2014 da disciplina de Metodologia da Pesquisa do curso de pós-graduação stricto sensu Mestrado em Computação Aplicada da UDESC pelas análises efetuadas durante o curso. Agradecemos à CAPES (Coordenação de Aperfeiçoamento de Pessoal de Nível Superior) pela concessão da bolsa de estudos para as alunas Adelaine Gelain e Marina Silva Fouto durante a realização do mestrado. 


\section{Referências}

[1] DENNING, P. J. Is computer science Science? Communications of the ACM. v. 48, n. 4, p. 27-31. 2005.

[2] PPINPECAP Programa de Pós-Graduação do Instituto Nacional de Pesquisa Espacial (INPE) em Computação Aplicada (CAP), São José dos Campos. Disponível em: <http://www.inpe.br/pos_graduacao/cursos/cap/>. Acesso em: 15 ago. 2014.

[3] Pós Graduação em Computação Aplicada da Universidade Estadual de Feira de Santana (UEFS), Feira de Santana. Disponível em: 〈http://pgca.uefs.br/area-de-concentracao-e-linhas-de-pesquisa〉. Acesso em: 17 ago. 2014.

[4] Pós Graduação em Computação Aplicada da Universidade do Estado de Santa Catarina (UDESC), Joinville. Disponível em: <http://www.cct.udesc.br/?id=342>. Acesso em: 15 ago. 2014.

[5] PPGCA - Programa de Pós-Graduação em Computação Aplicada, Universidade de Passo Fundo (UPF), Passo Fundo. Disponível em: <http://www.upf.br/ppgca>. Acesso em: 18 ago. 2014

[6] RBCA - Revista Brasileira de Computação Aplicada, UPF Editora; Passo Fundo-RS. Disponível em: <http://www.upf.br/seer/index.php/rbca>. Acesso em: 17 ago. 2014.

[7] DETROZ, J. P.; JASINSKI, M.G.; BOSSE, R.; BERLIM, T.H.; HOUNSELL, M.S. Virtual Reality Evolution in Brazil. A survey over the papers in Symposium on Virtual and Augmented Reality. In: XVI Symposium on Virtual and Augmented Reality, SVR 2014, Salvador, 2014.

[8] TICHY, W. F.; LUKOWICZ, P.; PRECHELT, L.; HEINZ, E. A. Experimental Evaluation in Computer Science: a Quantitative Study.Journal of Systems and Software 28 v.1, p. 9-18. 1995.

[9] WAINER, J.; BARSOTTINI, C. G. N.; LACERDA, D.; MARCO, L. R. M. Empirical Evaluation in Computer Science Research Published by ACM. Information and Software Technology. v. 51, 2009. p. 10811085.

[10] MORRISON, J; GEORGE, J. F. Exploring the software engineering component in MIS research. Communications of the ACM, v. 38, n.7, p. 80-91. 1995.

[11] WAZLAWICK, R. S. Metodologia de pesquisa para Ciência da Computação. 6a. impressão. Rio de Janeiro: Elsevier, 2009.

[12] EDEN, A. H. Three paradigms of Computer Science. Minds and machines. Special Issue on the Philosophy of Computer Science, v. 17, n. 2, p. 135-167. 2007.

[13] CLAVER, E.; GONZALEZ, R.; LOPIS, J. An analysis of research in information systems (1981-1997). Information \& Management, v. 37, p. 181-195. 2000.

[14] MAGALHÃES, C. V.; SANTOS, R. E.; NETO, J. S. C.; SOUZA, E. P. Tradição nos estilos de pesquisa em Educação a Distância: uma análise a partir das publicações do SBIE. In: WORKSHOP DE INFORMÁTICA NA ESCOLA, v. 1, p. 1604-1607, Anais... 2011.

[15] RAPOSO, A.; SOARES, L.; KELNER, J.; TEICHRIEB, V. A Journey Through Virtual and Augmented Reality: reviewing the SVR Symposia from 2004 to 2008. Revista de Informática Teórica e Aplicada, v.15, n. 3, p. 9-24. 2008 . 